\title{
Development of palm-based neopentyl glycol diester as dielectric fluid and its thermal aging performance
}

\begin{abstract}
The potential of palm-based neopentyl glycol diester as dielectric insulating fluid was investigated. The details of the transesterification of high oleic palm oil methyl ester (POME) with neopentyl glycol (NPG) with the final product yield of more than $90 \mathrm{wt} \%$ of NPG diester were discussed. The thermal aging performance of NPG diester was compared with conventional mineral insulating oil at 90,110 and $130{ }^{\circ} \mathrm{C}$. This paper focused mainly in the effects of aging to chemical, physical and electrical properties of NPG diester. Apart from being fully biodegradable and non-toxic, the synthesized diesters exhibited high flashpoint and the breakdown voltage was comparable to mineral oil. The result indicated that throughout the aging period, NPG diester exhibited lower acid value than mineral oil and no significant change in viscosity was observed. The study on mechanical properties of insulating paper aged in NPG diester shows higher tensile strength than paper aged in mineral oil. The synthesized esters have shown great potential to be used as transformer oil.
\end{abstract}

Keyword: $\quad$ Breakdown voltage; Insulating oil; Transformers; Thermal aging; Kraft paper; Palmbased ester; Mineral oil 